\title{
A Unified Analysis of DC Link Current in Space-Vector PWM Drives
}

\author{
C.C. Chan, K.T. Chau, Y. Li, and D.T.W. Chan* \\ Department of Electrical \& Electronic Engineering, The University of Hong Kong, Pokfulam Road, Hong Kong \\ * Department of Electrical \& Communications Engineering, Hong Kong Technical College, Tsing Yi, Hong Kong
}

\begin{abstract}
A unified analysis of the DC link current in spacevector PWM drives, comprising induction motor, inverter and space-vector PWM scheme, is presented. The DC link current is investigated in a unified way, no matter the inverter is operating at normal or dead-time switching modes. The key is to introduce a dead-time vector, which is mathematically similar to the well-known space voltage vector, to express the DC link current and inverter output voltages into a unified form. Moreover, the formation of positive and negative spikes on the DC link current is discussed. In particular, the occurrence of negative spikes is mathematically formulated. The proposed unified approach is verified by means of both computer simulation and experimental results. The occurrence of positive and negative spikes is also verified by using circuitoriented and device-oriented computer simulations as well as experimental results.
\end{abstract}

\section{Introduction}

The DC link current of PWM inverter-fed AC motor drives has been of utmost importance for system protection, efficiency optimization and state identification. The characteristics of the DC link current have been discussed and analyzed using traditional PWM schemes [1]-[2]. However, these approaches are unnecessarily complicated and impractical for modern drive systems using space vector PWM schemes [3]-[4]. Nevertheless, they have been developed mainly for steady-state analysis, while the characteristic current during transient operation, which are of much significance, have seldom been investigated.

On the other hand, it is well known that a so-called deadtime interval is purposely employed to protect the power switches within the same inverter leg from shoot-through failure. The available studies of the effect of dead time has been mainly focused on the inverter output side, such as the distortion of output voltage/current and the oscillation of motor torque [5]. Although its effect on the input side, namely the DC link current, has been mentioned, their discussions are essentially qualitative [2].

It has also been well known that there are significant spikes on the inverter DC link current waveform. Since the DC link current essentially represents the power flow between the power source and the inverter drive, these current spikes indicate that there are sudden changes of power flow, either powering or regenerating, depending on positive or negative spikes. It is apt to consider that these current spikes are due to the switching transients of power devices, especially the reverse recovery effect of freewheeling diodes. However, are all these current spikes exclusively caused by the switching transients? Does the existence of dead time cause any spikes on the DC link current? Can the effect of these current spikes be included in the system equation of the whole drive? To the best knowledge of the authors, these questions have neither been mentioned nor explained yet.

It is the purpose of this paper to develop a new approach to the analysis of the DC link current in PWM inverter-fed $\mathrm{AC}$ motor drives in the presence of dead time. Thus, the entire drive system, including the inverter and motor, can be investigated as a whole, no matter operating in the normal or dead-time mode, during steady-state and transient conditions. Moreover, the aforementioned questions will be discussed, especially the occurrence of negative spikes on the DC link current due to the existence of dead time.

In Section 2, a novel concept, namely the dead-time vector (DTV), will be introduced which possesses the same mathematical form but different physical nature compared to the well known space voltage vector (SVV). Hence, the effect of dead time can be incorporated into the system equation of the whole drive. Section 3 will be devoted to mathematically formulate the occurrence of negative current spikes. In Sections 4 and 5, computer simulations, including circuitoriented simulation based on $\mathrm{C}++$ and device-oriented simulation based on SPICE, and experimental results are given to verify the proposed approach and the formation of both positive and negative current spikes.

\section{Unified Approach}

By newly introducing the DTV to the SVV based system equation of the entire drive, the analysis of the system performance in both normal and dead-time operating modes can be unified. A voltage-source space-vector PWM inverterfed induction motor drive is used to exemplify the proposed unified approach. As shown in Fig. 1, $I_{d c}$ is the instantaneous DC link current, $I_{u}, I_{v}$ and $I_{w}$ are the instantaneous threephase upper leg currents, and $I_{a}, I_{b}$ and $I_{c}$ are the instantaneous three-phase winding currents.

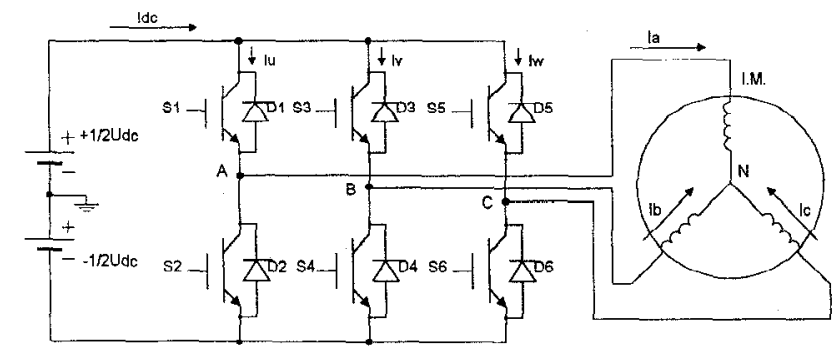

Fig. 1: 3-phase PWM inverter with induction motor load 


\subsection{Space voltage vectors}

During the normal operating mode, there are eight switching states which can be expressed in terms of the well known SVV, namely $\left[\begin{array}{lll}S_{a} & S_{b} & S_{c}\end{array}\right]^{\mathrm{T}}$. These vectors, with components either zero or unity, have the physical meaning that $S_{p}=0(p=a, b, c)$ when the upper leg switch of phase $p$ is off and the lower one is on, and vice versa. Hence, the threephase terminal voltages can be written as:

$\left\{\begin{array}{l}U_{a}=\frac{1}{2} U_{d c}\left(2 S_{a}-1\right) \\ U_{b}=\frac{1}{2} U_{d c}\left(2 S_{b}-1\right) \\ U_{c}=\frac{1}{2} U_{d c}\left(2 S_{c}-1\right)\end{array}\right.$

Projecting the terminal voltages given by (1) onto the $\alpha-\beta$ plane shown in Fig. 2, the resulting orthogonal voltage components can be expressed in terms of the SVV:

$\left[\begin{array}{l}U_{\alpha} \\ U_{\beta}\end{array}\right]=\sqrt{\frac{2}{3}}\left[\begin{array}{ccc}1 & -\frac{1}{2} & -\frac{1}{2} \\ 0 & \frac{\sqrt{3}}{2} & -\frac{\sqrt{3}}{2}\end{array}\right]\left[\begin{array}{l}S_{a} \\ S_{b} \\ S_{c}\end{array}\right] U_{d c}$

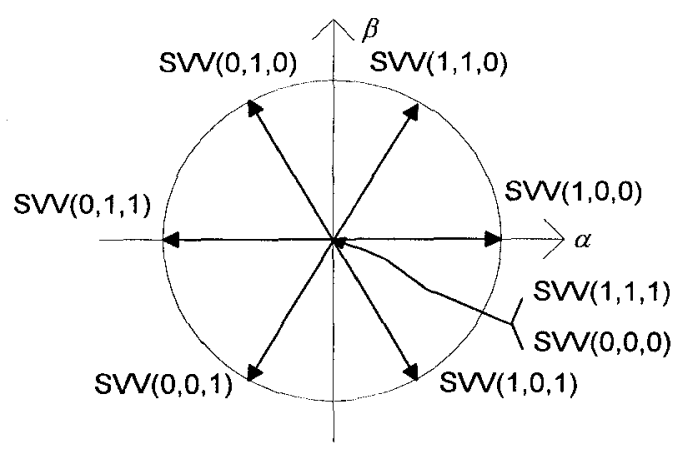

Fig. 2: Voltage vectors and space sectors

Given the SVV, the voltage vector can be obtained from (2), hence the winding currents can be calculated simply according to the mathematical model of the motor. To derive the relationship between the $\mathrm{DC}$ link current and those winding currents, the upper leg currents are used as intermediates. When $S_{a}=1$, the winding terminal point A is connected to the positive supply rail, and the current flows through the upper leg of the inverter (either $S_{1}$ or $D_{1}$ conducts, depending on $I_{a}>0$ or $I_{a}<0$ ), thus $I_{u}=I_{a}$. On the contrary, when $S_{a}=0$, the current flows through the lower leg (either $D_{4}$ or $S_{4}$ conducts, depending on $I_{a}>0$ or $I_{a}<0$ ) instead of the upper one, thus $I_{u}=0$. The situations for phases $\mathrm{B}$ and $\mathrm{C}$ are similar. Hence, the relationship between the leg and winding currents can be written as:

$\left\{\begin{array}{l}I_{u}=I_{a} S_{a} \\ I_{v}=I_{b} S_{b} \\ I_{w}=I_{c} S_{c}\end{array}\right.$

Since the DC link current is always equal to the sum of three upper leg currents, namely $I_{d c}=I_{u}+I_{v}+I_{w}$, the expression of the DC link current can then be obtained as:

$I_{d c}=I_{a} S_{a}+I_{b} S_{b}+I_{c} S_{c}$

\subsection{Dead-time vectors}

During the dead-time operating mode, both of the power switches along the same leg are turned off, while the winding current is flowing through a freewheeling diode as dictated by the current direction. Fig. 3 shows the corresponding freewheeling process of phase $A$ in which there are two different freewheeling paths, depending on the direction/polarity of $I_{a}$.

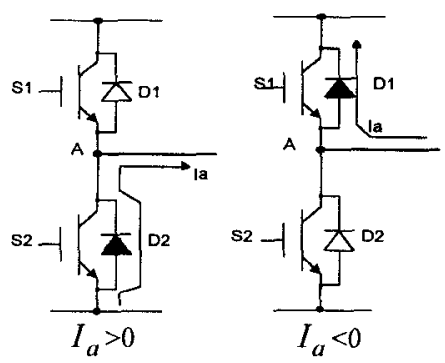

Fig. 3: Current flow during dead-time interval

When $I_{a}>0$, the winding current is freewheeling via the diode $D_{2}$ in the lower leg. Thus, the terminal $\mathrm{A}$ is connected to the negative supply rail, and there is no current flowing through the upper leg, leading to $U_{a}=-U_{d c} / 2$ and $I_{u}=0$. On the contrary, when $I_{a}<0$, the winding current is freewheeling via the diode $D_{1}$ in the upper leg. Thus, the terminal $\mathrm{A}$ is connected to the positive supply rail, and the upper leg current is equal to the winding current, leading to $U_{a}=U_{d c} / 2$ and $I_{u}=I_{a}$. In order to express the terminal voltage and upper leg current irrespective to the polarity of $I_{a}$, a bi-value function $D_{a}$ is newly defined which equals zero for $I_{a}>0$ and unity for $I_{a}<0$, hence:

$U_{a}=\frac{1}{2} U_{d c}\left(2 D_{a}-1\right)$

$I_{u}=I_{a} D_{a}$

The bi-value functions for phases $B$ and $C$ can similarly be defined, and all these functions are generalized as:

$D_{p}=\left\{\begin{array}{lll}0 & \text { when } & I_{p}>0 \\ 1 & \text { when } & I_{p}<0\end{array} \quad\right.$ where $p=a, b, c$

The corresponding terminal voltages and upper leg currents for phases $B$ and $C$ are given by:

$U_{b}=\frac{1}{2} U_{d c}\left(2 D_{b}-1\right)$

$I_{v}=I_{b} D_{b}$

$U_{c}=\frac{1}{2} U_{d c}\left(2 D_{c}-1\right)$

$I_{w}=I_{c} D_{c}$

It should be noted that the above terminal voltages and upper leg current, given by (5), (8), (10) and (6), (9), (11) respectively, have the unified form as those voltages and currents at the normal operating mode as given by (1) and (3) respectively. The sole difference is the change of $S_{p}$ to $D_{p}$. Although $S_{p}$ and $D_{p}$ have different physical meanings (the former representing the on/off of the power switch whereas the latter representing the polarity of the winding current), they 
have the same mathematical form. Therefore, $S_{p}$ and $D_{p}$ can work together to form a new vector, namely the DTV.

Theoretically, there are 27 possible combinations of switching states in a three-phase inverter. Subtracting those 8 combinations for the SVV during the normal mode of operation, the remaining 19 combinations are for the DTV during the dead-time operating mode. To facilitate the discussion and derivation, these 19 DTV combinations can be classified into three cases:

Case 1. Only one phase changes its state while the other two phases keep unchanged.

Case 2. Two phases simultaneously change their states while the remaining one keeps unchanged.

Case 3. All three phases change their states simultaneously. However, practically, the Case 3 is absent in available PWM switching schemes and will not be discussed in details.

For the Case 1 , considering that phase $A$ involves the change of its state while both phases $B$ and $C$ have no change, the terminal voltages and their projected values onto the $\alpha-\beta$ plane can be expressed as:

$\left\{\begin{array}{l}U_{a}=\frac{1}{2} U_{d c}\left(2 D_{a}-1\right) \\ U_{b}=\frac{1}{2} U_{d c}\left(2 S_{b}-1\right) \\ U_{c}=\frac{1}{2} U_{d c}\left(2 S_{c}-1\right)\end{array}\right.$

$\left[\begin{array}{l}U_{\alpha} \\ U_{\beta}\end{array}\right]=\sqrt{\frac{2}{3}}\left[\begin{array}{rrr}1 & -\frac{1}{2} & -\frac{1}{2} \\ 0 & \frac{\sqrt{3}}{2} & -\frac{\sqrt{3}}{2}\end{array}\right]\left[\begin{array}{l}D_{a} \\ S_{b} \\ S_{c}\end{array}\right] U_{d c}$

where $\left[\begin{array}{lll}D_{a} & S_{b} & S_{c}\end{array}\right]^{\mathrm{T}}$ is the corresponding DTV. Similar to that derived for (4), the expression of the DC link current can be obtained from the summation of all upper leg currents:

$I_{d c}=I_{a} D_{a}+I_{b} S_{b}+I_{c} S_{c}$

Similarly, for the Case 2, considering that both phases A and $B$ involve changing their states while phase $C$ keeps unchanged, the terminal and projected voltages can be expressed as:

$\left\{\begin{array}{l}U_{a}=\frac{1}{2} U_{d c}\left(2 D_{a}-1\right) \\ U_{b}=\frac{1}{2} U_{d c}\left(2 D_{b}-1\right) \\ U_{c}=\frac{1}{2} U_{d c}\left(2 S_{c}-1\right)\end{array}\right.$
$\left[\begin{array}{l}U_{\alpha} \\ U_{\beta}\end{array}\right]=\sqrt{\frac{2}{3}}\left[\begin{array}{ccc}1 & -\frac{1}{2} & -\frac{1}{2} \\ 0 & \frac{\sqrt{3}}{2} & -\frac{\sqrt{3}}{2}\end{array}\right]\left[\begin{array}{l}D_{a} \\ D_{b} \\ S_{c}\end{array}\right] U_{d c}$

where $\left[\begin{array}{lll}D_{a} & D_{b} & S_{\mathrm{c}}\end{array}\right]^{\mathrm{T}}$ is the corresponding DTV. The DC link current can then be expressed as:

$$
I_{d c}=I_{a} D_{a}+I_{b} D_{b}+I_{c} S_{c}
$$

Since the DTV in (13) or (16) has the unified form as the SVV in (2), the inverter voltages and currents can be analyzed as a whole, no matter operating in normal or dead-time mode. This unified approach is particularly important and useful for performance evaluation, digital simulation and system control of the entire PWM drive.

\section{Formulation of Negative Spikes}

As mentioned previously, there are significant spikes on the DC link current. The positive spikes are mainly due to the reverse recovery of freewheeling diodes which will not be further discussed. On the other hand, the negative spikes which occur only but not usually at the dead-time intervals will be discussed by using the newly proposed DTV approach.

As shown in Fig. 4, the DTV operating in the dead-time interval $T_{d}$ is inserted between the transition from a SVV, $\left[\begin{array}{lll}S_{a, n} & S_{b, n} & S_{c, n}\end{array}\right]^{\mathrm{T}}$, operating in the normal interval $T_{n}$ to another SVV, $\left[S_{a, n+1} S_{b, n+1} S_{c, n+1}\right]^{T}$, in $T_{n+1}$. Since $T_{d}$ is much shorter than $T_{n}$ and $T_{n+1}$, the negative current spike means that $I_{d c, \text { dead }}$ at $T_{d}$ is smaller than $I_{d c, n}$ at $T_{n}$ and $I_{d c, n+1}$ at $T_{n+1}$, respectively before and after $T_{d}$, and is either negative or zero. Hence, three criteria for the formation of these negative current spikes can be expressed as:

$$
\begin{aligned}
& I_{d c, \text { dead }}<I_{d c, n} \\
& I_{d c, \text { dead }}<I_{d c, n+1} \\
& I_{d c, \text { dead }} \leq 0
\end{aligned}
$$

Since the Case 3 that all phases changing their states simultaneously is generally absent in PWM switching schemes for $\mathrm{AC}$ motor control, the discussion and derivation of the occurrence of negative current spikes will be based on the Cases 1 and 2 .

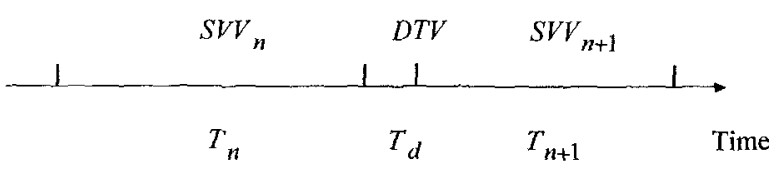

Fig. 4: Transition of switching states

\subsection{Case 1 (change in one phase)}

Considering that only phase A changing its state, the SVV changes from $\left[\begin{array}{lll}S_{a, n} & S_{b, n} & S_{c, n}\end{array}\right]^{\mathrm{T}}$ to $\left[\begin{array}{lll}S_{a, n+1} & S_{b, n} & S_{c, n}\end{array}\right]^{\mathrm{T}}$. Thus, the DC link currents at, before and after $T_{d}$ are expressed as:

$$
\begin{aligned}
& I_{d c, \text { dead }}=I_{a} D_{a}+I_{b} S_{b, n}+I_{c} S_{c, n} \\
& I_{d c, n}=I_{a} S_{a, n}+I_{b} S_{b, n}+I_{c} S_{c, n} \\
& I_{d c, n+1}=I_{a} S_{a, n+1}+I_{b} S_{b, n}+I_{c} S_{c, n}
\end{aligned}
$$

Since $S_{a, n}, S_{a, n+1}$ and $D_{a}$ are either zero or unity while $S_{a, n} \neq S_{a, n+1}$, there are only two possible results, either $D_{a}=S_{a, n}$ or $D_{a}=S_{a, n+1}$, leading to obtain either $I_{d c, \text { dead }}=I_{d c, n}$ using (19) and (20), or $I_{d c, \text { dead }}=I_{d c, n+1}$ using (20) and (21). These two results dissatisfy the criteria in (18a) and (18b), respectively. Therefore, the case with only one phase involving the change of state will not cause a negative current spike at the dead-time interval.

\subsection{Case 2 (changes in two phases)}

Considering that both phases $\mathrm{A}$ and $\mathrm{B}$ changing their states, the SVV changes from $\left[S_{a, n} S_{b, n} S_{c, n}\right]^{\mathrm{T}}$ to $\left[\begin{array}{lll}S_{a, n+1} & S_{b, n+1} & S_{c, n}\end{array}\right]^{\mathrm{T}}$. The corresponding DC link currents at, before and after $T_{d}$ are expressed as: 
$I_{d c, \text { dead }}=I_{a} D_{a}+I_{b} D_{b}+I_{c} S_{c, n}$

$I_{d c, n}=I_{a} S_{a, n}+I_{b} S_{b, n}+I_{c} S_{c, n}$

$I_{d c, n+1}=I_{a} S_{a, n+1}+I_{b} S_{b, n+1}+I_{c} S_{c, n}$

By using (22)-(24), when $\left[\begin{array}{ll}D_{a} & D_{b}\end{array}\right]^{\mathrm{T}}=\left[\begin{array}{ll}S_{a, n} & S_{b, n}\end{array}\right]^{\mathrm{T}}$, $I_{d c, \text { dead }}=I_{d c, n}$, implying that the criterion in (18a) can not be satisfied. Similarly, when $\left[\begin{array}{ll}D_{a} & D_{b}\end{array}\right]^{\mathrm{T}}=\left[\begin{array}{ll}S_{a, n+1} & S_{b, n+1}\end{array}\right]^{\mathrm{T}}$, $I_{d c, \text { dead }}=I_{d c, n+1}$, dissatisfying the criterion in (18b). Hence, for both of the above conditions, there is no negative current spike at the dead-time interval. On the contrary, both the criteria in (18a) and (18b) can be satisfied when fulfilling the following inequality:

$\left[\begin{array}{ll}D_{a} & D_{b}\end{array}\right]^{\mathrm{T}} \neq\left[\begin{array}{ll}S_{a, n} & S_{b, n}\end{array}\right]^{\mathrm{T}} \neq\left[\begin{array}{ll}S_{a, n+1} & S_{b, n+1}\end{array}\right]^{\mathrm{T}}$

For exemplification, considering the condition that $\left[\begin{array}{ll}D_{a} & D_{b}\end{array}\right]^{\mathrm{T}}=\left[\begin{array}{ll}0 & 0\end{array}\right]^{\mathrm{T}}, \quad\left[\begin{array}{ll}S_{a, n} & S_{b, n}\end{array}\right]^{\mathrm{T}}=\left[\begin{array}{ll}0 & 1\end{array}\right]^{\mathrm{T}} \quad$ and $\left[\begin{array}{ll}S_{a, n+1} & S_{b, n+1}\end{array}\right]^{\mathrm{T}}=\left[\begin{array}{ll}1 & 0\end{array}\right]^{\mathrm{T}}, I_{a}>0$ and $I_{b}>0$ are resulted by using (7), and then the following results can be generated:

$$
\begin{aligned}
& I_{a} D_{a}+I_{b} D_{b}=0 \\
& I_{a} S_{a, n}+I_{b} S_{b, n}=I_{b}>0 \\
& I_{a} S_{a, n+1}+I_{b} S_{b, n+1}=I_{a}>0
\end{aligned}
$$

By substituting (26)-(28) into (22)-(24), it can be easily deduced that $I_{d c \text {,dead }}<I_{d c, n}$ and $I_{d c, \text { dead }}<I_{d c, n+1}$. Similarly, the other combinations fulfilling the inequality in (25) can be determined. As listed in Table 1, there are totally eight combinations satisfying the necessary criteria in (18a) and (18b) for generating negative current spikes.

Moreover, the value of $I_{d c \text {,dead }}$ needs to be calculated to confirm whether the criterion in $(18 \mathrm{c})$ can be satisfied. By substituting the values of $D_{a}, D_{b}$ and $S_{c, n}$ into (22) and then making use of the constraint $I_{a}+I_{b}+I_{c}=0$, the corresponding value of $I_{d c \text {,dead }}$ can be determined. For exemplification, considering that $D_{a}=0, D_{b}=0$ and $S_{c, n}=1$, $I_{a}>0$ and $I_{b}>0$ are resulted by using (7); hence, $I_{d c, \text { dead }}=I_{c}=-\left(I_{a}+I_{b}\right)<0$ can be deduced by using (22) and the constraint. As shown in Table 2, under all possible combinations of $D_{a}, D_{b}$ and $S_{c, n}$, independent of the polarities of the winding currents $I_{a}, I_{b}$ and $I_{c}$, it can be found that $I_{d c \text {,dead }} \leq 0$, satisfying the criterion in (18c).

Therefore, when phases $\mathrm{A}$ and $\mathrm{B}$ simultaneously change their states under the condition given by (25), there is a negative spike on the DC link current during the dead-time interval. Also, the magnitude of this negative current spike is equal to the winding current in phase $\mathrm{A}, \mathrm{B}$ or $\mathrm{C}$, namely $I_{a}$, $I_{b}$ or $I_{c}$.

\section{Computer Simulations}

A voltage-source space-vector PWM inverter-fed induction motor drive [6] is adopted for the following computer simulations.

\subsection{Circuit-oriented simulation based on $\mathrm{C}++$}

The purpose of circuit-oriented simulation based on $\mathrm{C}++$ is to investigate the performance of the whole drive system in the presence of dead time, during both transient and steady states. The power switches and diodes are assumed ideal and all the values are presented in per unit.

The waveforms of $I_{d c}$ and $I_{a}$ at $50 \mathrm{~Hz}$ for one fundamental operation period $(20 \mathrm{~ms})$ are shown in Fig. 5. Within the fundamental period of $20 \mathrm{~ms}$, it can be found that there are six pulses in the envelop of $I_{d c}$. It is because the whole $360^{\circ}$ plane is divided into six sectors by the voltage vectors as shown in Fig. 2.

The waveforms of instantaneous $I_{a}$ and $I_{d c}$ when the inverter fed induction motor is being started-up from standstill at $50 \mathrm{~Hz}$ are shown in Fig. 6. There are both positive and negative values at the instantaneous DC link current during transient process because of the relatively low and changing power factor of the induction motor when it is being started-up from standstill.

To illustrate the effect of dead time, waveforms of $I_{d c}$ within one fundamental operation period during steady state are shown in Fig. 7, with and without dead time being considered. If dead time is not considered, there is definitely no negative spike at $I_{d c}$. On the contrary, if dead time is considered as $T_{d}=10 \mu \mathrm{s}$, significant negative spikes are found on $I_{d c}$.

Table 1: Possible combinations satisfying first two criteria for negative current spikes

\begin{tabular}{ccccccccc}
\hline$\left[S_{a, n}\right.$ & {$\left[S_{a, n+1}\right.$} & {$\left[D_{a}\right.$} & $I_{a}$ & $I_{b}$ & $I_{a} S_{a, n}$ & $I_{a} S_{a, n+1} I_{a} D_{a}$ & $\mathrm{OK} ?$ \\
$\left.S_{b, n}\right]$ & $\left.S_{b, n+1}\right]$ & $\left.D_{b}\right]$ & & & + & + & + & \\
\hline$[0,0]$ & {$[1,1]$} & {$[0,1]$} & $>0$ & $<0$ & 0 & $I_{a}+I_{b}$ & $I_{b}$ & $\mathrm{Y}$ \\
{$[0,0]$} & {$[1,1]$} & {$[1,0]$} & $<0$ & $>0$ & 0 & $I_{a}+I_{b}$ & $I_{a}$ & $\mathrm{Y}$ \\
{$[1,1]$} & {$[0,0]$} & {$[0,1]$} & $>0$ & $<0$ & $I_{a}+I_{b}$ & 0 & $I_{b}$ & $\mathrm{Y}$ \\
{$[1,1]$} & {$[0,0]$} & {$[1,0]$} & $<0$ & $>0$ & $I_{a}+I_{b}$ & 0 & $I_{a}$ & $\mathrm{Y}$ \\
{$[0,1]$} & {$[1,0]$} & {$[1,1]$} & $<0$ & $<0$ & $I_{b}$ & $I_{a}$ & $I_{a}+I_{b}$ & $\mathrm{Y}$ \\
{$[0,1]$} & {$[1,0]$} & {$[0,0]$} & $>0$ & $>0$ & $I_{b}$ & $I_{a}$ & 0 & $\mathrm{Y}$ \\
{$[1,0]$} & {$[0,1]$} & {$[1,1]$} & $<0$ & $<0$ & $I_{a}$ & $I_{b}$ & $I_{a}+I_{b}$ & $\mathrm{Y}$ \\
{$[1,0]$} & {$[0,1]$} & {$[0,0]$} & $>0$ & $>0$ & $I_{a}$ & $I_{b}$ & 0 & $\mathrm{Y}$ \\
\hline
\end{tabular}

Table 2: $D_{a}, D_{b}$ and $S_{c, n}$ satisfying $I_{d c, d e a d} \leq 0$

\begin{tabular}{lllllll}
\hline$S_{c, n}$ & $D_{a}$ & $D_{b}$ & $I_{a}$ & $I_{b}$ & $I_{d c, \text { dead }}$ & $I_{d c, \text { dead }}<0 ?$ \\
\hline 0 & 0 & 0 & $>0$ & $>0$ & 0 & \\
0 & 0 & 1 & $>0$ & $<0$ & $I_{b}$ & $\mathrm{Y}$ \\
0 & 1 & 0 & $<0$ & $>0$ & $I_{a}$ & $\mathrm{Y}$ \\
0 & 1 & 1 & $<0$ & $<0$ & $I_{a}+I_{b}$ & $\mathrm{Y}$ \\
1 & 0 & 0 & $>0$ & $>0$ & $-\left(I_{a}+I_{b}\right)$ & $\mathrm{Y}$ \\
1 & 0 & 1 & $>0$ & $<0$ & $-I_{a}$ & $\mathrm{Y}$ \\
1 & 1 & 0 & $<0$ & $>0$ & $-I_{b}$ & $\mathrm{Y}$ \\
1 & 1 & 1 & $<0$ & $<0$ & 0 & \\
\hline
\end{tabular}




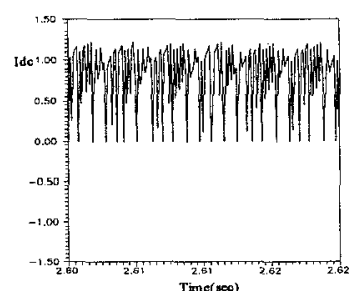

(a)

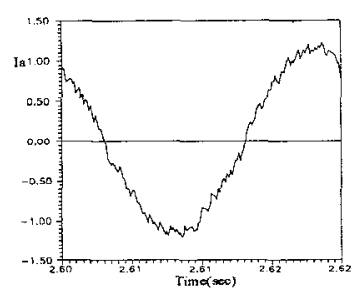

(b)
Fig. 5: Steady state waveforms without dead time (a) DC link current (b) Winding current

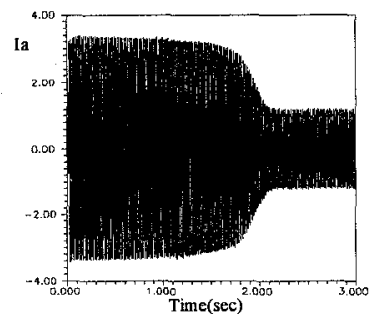

(a)

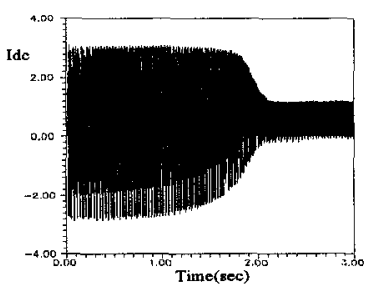

(c)

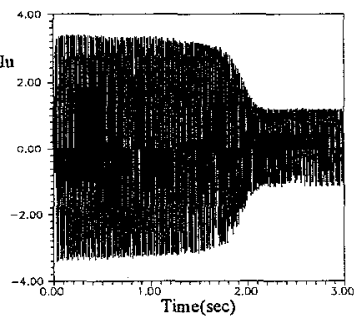

(b)

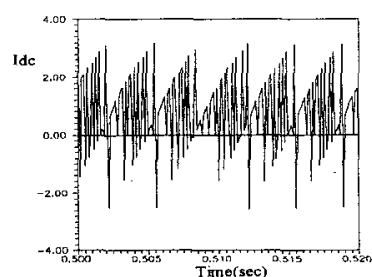

(d)
Fig. 6: Start-up transient waveforms without dead time

(a) Winding current (b) Leg current

(c) DC link current (d) Zoomed DC link current

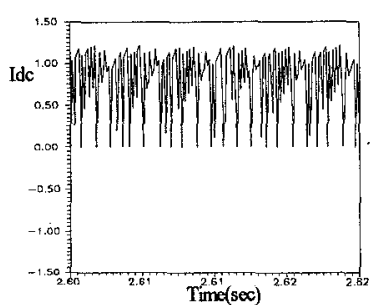

(a)

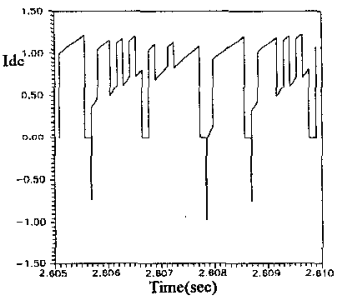

(c)

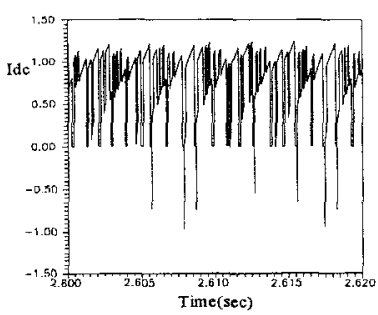

(b)

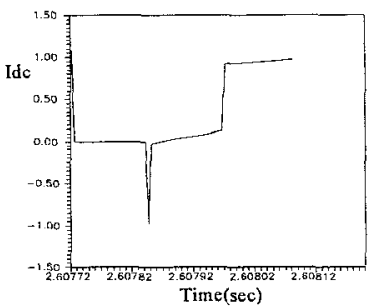

(d)
Fig. 7: Negative spikes on DC link current (a) Without dead time (b) With dead time (c) With dead time (zoomed) (d) With dead time (close-up)

\subsection{Device-oriented simulation based on SPICE}

In order to fully analyze current spikes in this practical system, device-oriented simulation based on SPICE has been performed, in which the characteristics of power devices and diodes can be taken into account [7].

Firstly, the simulation is carried out in the absence of dead time. The resulting waveforms of $I_{d c}, I_{u}$ and $I_{a}$ are shown in Fig. 8. As expected, no negative spikes can be found on the waveform of $I_{d c}$. Moreover, there are obvious positive spikes on the waveforms of $I_{d c}$ and $I_{u}$, which are caused by the reverse recovery effect of freewheeling diodes. When a switch is being turned on and the conducting diode at the same leg is being blocked off by this turn on, because of the reverse recovery effect of diode, this leg is in fact short-circuited at this moment, leading to the occurrence of a positive current spike.

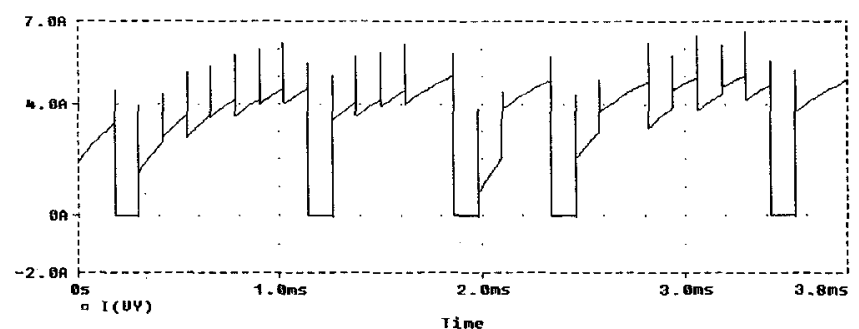

(a)

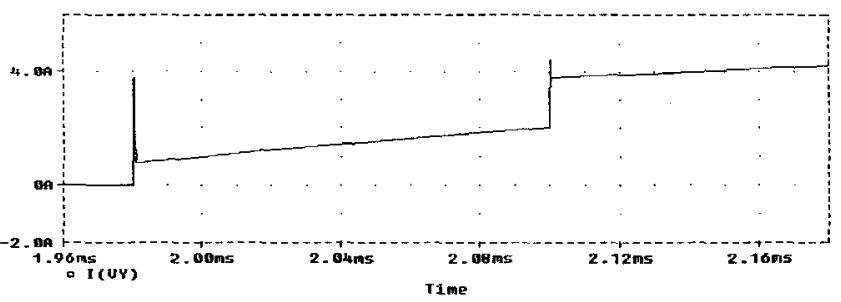

(b)

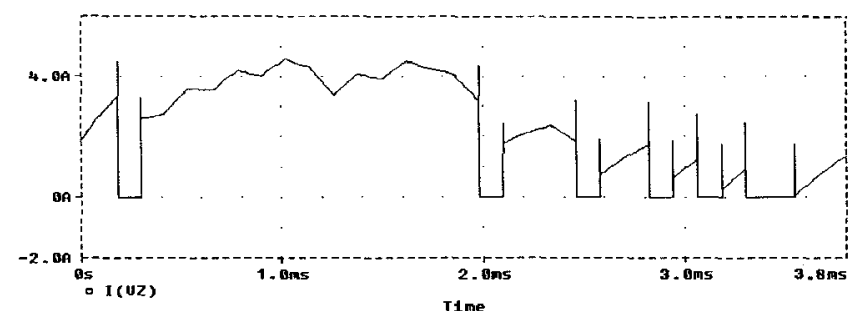

(c)

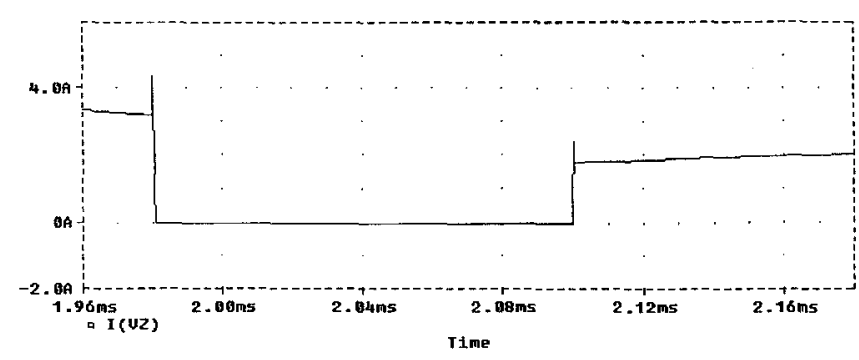

(d)

Fig. 8: SPICE simulation waveforms without dead time (a) DC link current (b) Zoomed DC link current (c) Upper leg current (d) Zoomed upper leg current 
Secondly, dead time is considered and the resulting waveforms of $I_{d c}$ and $I_{u}$ are shown in Fig. 9. It can be found that, with the introduction of dead time, there are significant negative spikes on the waveform of $I_{d c}$. As expected, there is no negative spike on the waveform of $I_{n}$, even though the reverse-recovery-effect-caused positive current spikes still exist. It should be noted that the time width of these negative spikes is equal to $10 \mu \mathrm{s}$, namely the preset dead time duration. A.lso, these negative spikes do not always occur in all subcycles.

\section{Experimental Results}

As used previously for computer simulations, the same set of voltage-source space-vector PWM inverter-fed induction motor drive is adopted for performing the following experimental verification.

The experimental waveform of the DC link current for one fundamental period $(20 \mathrm{~ms})$ is shown in Fig. 10. Fig. 11 shows the measured waveforms of DC link current and its corresponding inverter upper leg current. It can be observed that there are both negative and positive spikes on the measured DC link current waveform while there is only a positive spike on the measured leg current. It verifies that the negative current spike is not due to the switching transient of power devices such as reverse recovery of diodes. Moreover, the measured negative spike shows that its duration closely agrees with the dead-time interval of $10 \mu \mathrm{s}$.

Furthermore, Fig. 12 verifies that there is no negative current spike on $I_{d c}$ when the previously defined criteria are not satisfied during the dead-time mode of operation while the positive spike caused by reverse recovery of diodes is independent of inverter operation. It is obviously that the experimental results agree well with those simulation results.

\section{Conclusion}

With the introduction of the DTV concept, the DC link current of a space-vector PWM inverter-fed induction motor drive has been investigated by a unified approach, no matter operating in the normal or dead-time mode. Both steady-state and transient analyses have been carried out. It has been found that the negative spikes on the DC link current are caused by the inevitable dead-time intervals, and these negative current spikes essentially influence the stability of the drive system. Moreover, the occurrence of these negative spikes has been analytically formulated. Although the derivation and analysis have been based on the space-vector PWM inverter-fed induction motor drive, the approach can readily be applied to any $\mathrm{AC}$ motor drives using other PWM schemes and motors.

\section{Acknowledgment}

This work was supported and funded in part by the Committee on Research and Conference Grants, the University of Hong Kong.

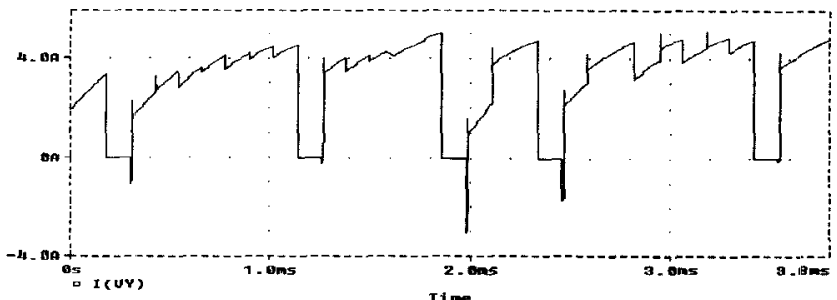

(a)

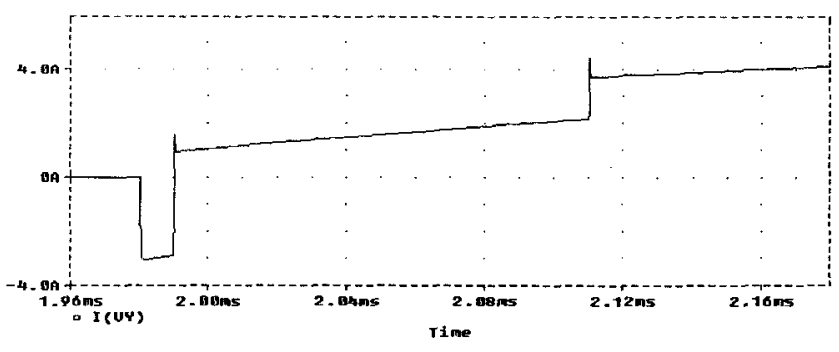

(b)

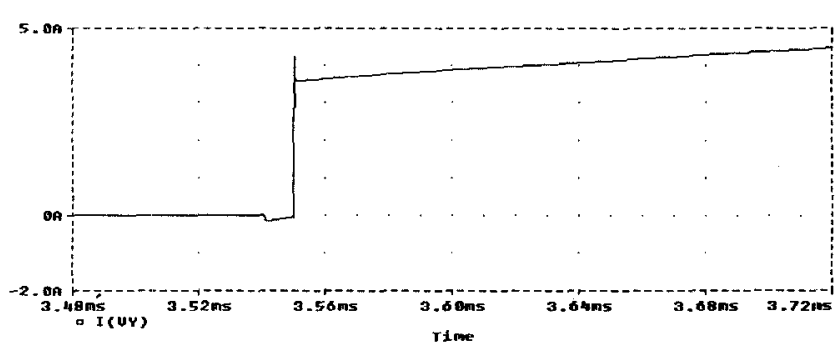

(c)

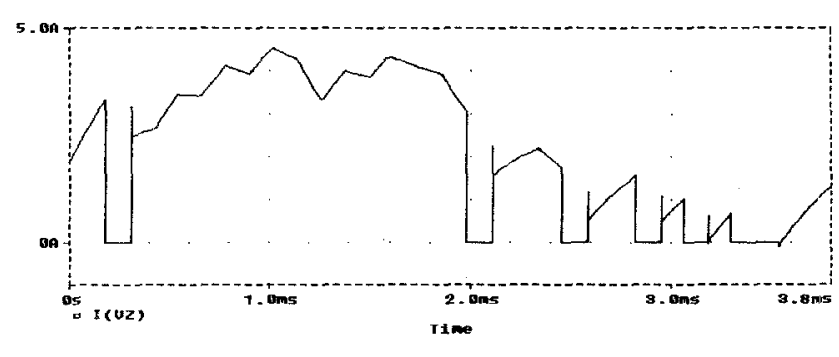

(d)

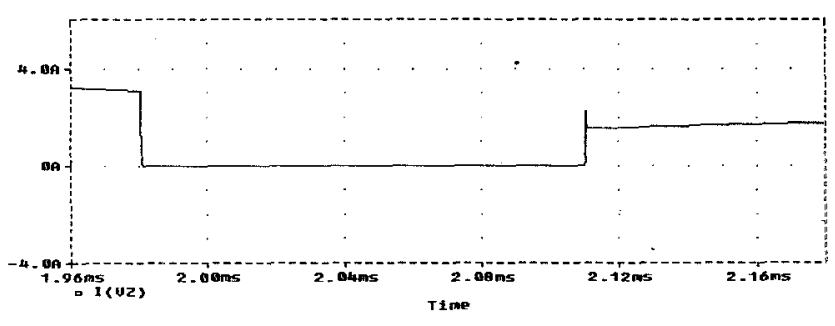

(e)

Fig. 9: SPICE simulation waveforms with dead time (a) DC link current (b) Zoomed DC link current with one negative spike (c) Zoomed DC link current without negative spike (d) Upper leg current (e) Zoomed upper leg current 


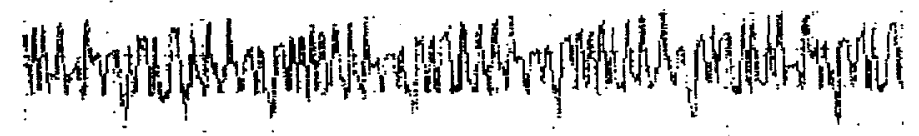

Fig. 10: Measured waveform of DC link current over $20 \mathrm{~ms}$

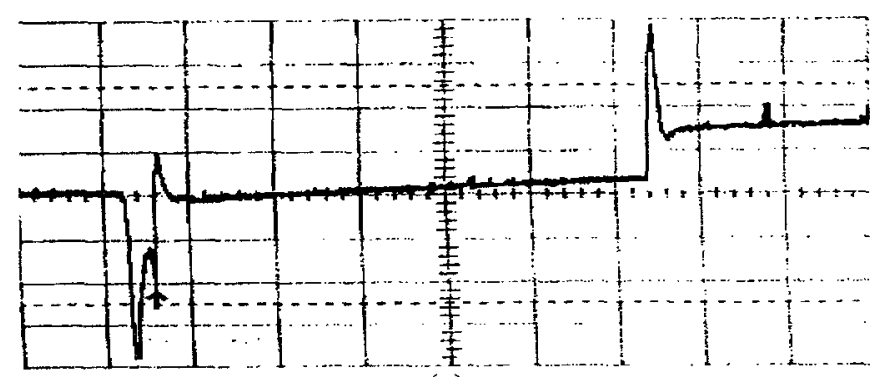

(a)

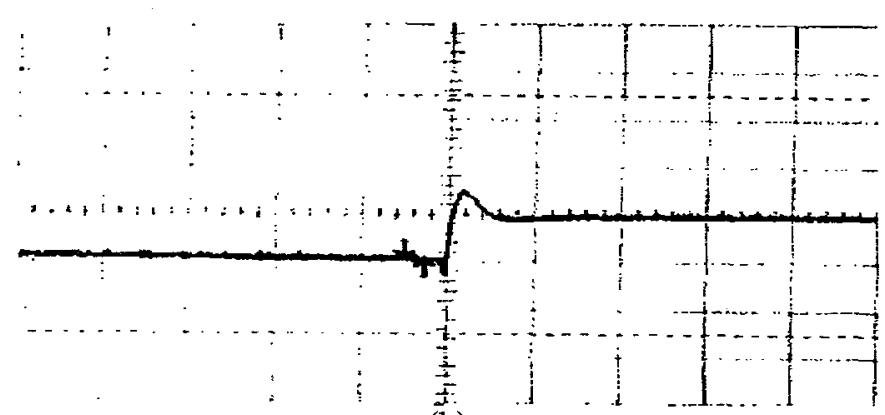

(b)

Fig 11: Measured current waveforms with negative spike

(a) DC link current $(20 \mu \mathrm{s} / \mathrm{div})$

(b) Upper leg current (10 $\mu \mathrm{s} /$ div $)$

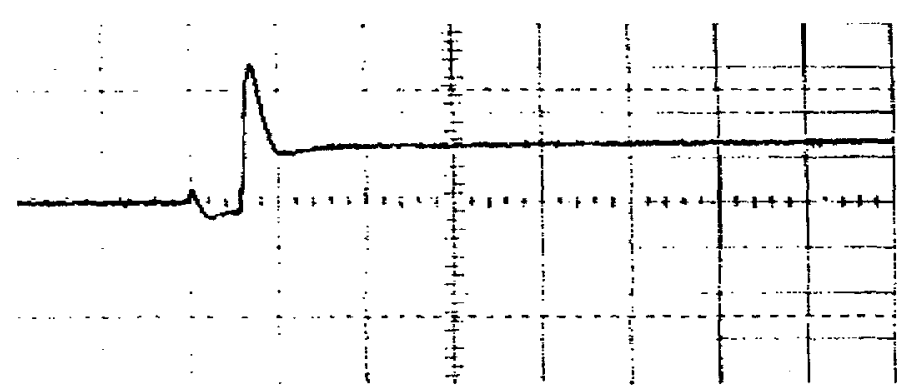

Fig. 12: Measured DC link current waveform without negative spike $(10 \mu \mathrm{s} / \mathrm{div})$

\section{References}

[1] P.D. Evans and R.J. Hill-Cottingham, "DC link current in PWM inverters," IEE Proceedings - B, vol. 133, 1983, pp. 217-224

[2] T.C. Green and B.W. Williams, "Derivation of motor linecurrent waveforms from the DC-link current of an inverter," IEE Proceedings - B, vol. 136, 1989, pp. 196204

[3] I. Takahashi and T. Noguchi, "A new quick-response and high efficiency control strategy of an induction motor," IEEE Transactions on Industry Applications, vol. 22, 1986, pp. 820-827

[4] T.G. Habetler and F. Profumo, "Direct torque control of induction machines using space vector modulation," In Proceedings of the IEEE Industry Applications Society Annual Meeting, 1991, pp. 428-436

[5] R.B. Sepe and J.H. Lang, "Inverter nonlinearities and discrete-time vector current control," IEEE Transactions on Industry Applications, vol. 30, 1994, pp. 62-70

[6] Y. Li, L. Huang and F. Li, "All digital space vector PWM induction motor drive based on flux control," In Proceedings of the IEEE TENCON, vol. 5, 1993, pp. 598601

[7] H. Rashid, SPICE for Power Electronics and Electric Power, Prentice-Hall, Inc., 1993 